\title{
Statistiques officielles et évolution des valeurs sociales : le cas de la personne repère dans les recensements canadiens de 1981 et de 1986 \\ OFFICIAL STATISTICS AND CHANGING SOCIAL VALUES ESTADISTICAS OFICIALES Y EVOLUCIÓN DE LOS VALORES SOCIALES : EL CASO DE LA PERSONA « REFERENCIA » EN LOS CENSOS CANADIENSES DE 1981 Y 1986.
}

\author{
Sylvia T. Wargon
}

Volume 18, numéro 2, automne 1989

Sous-dénombrement et estimation de population

URI : https://id.erudit.org/iderudit/010020ar

DOI : https://doi.org/10.7202/010020ar

Aller au sommaire du numéro

Éditeur(s)

Association des démographes du Québec

ISSN

0380-1721 (imprimé)

1705-1495 (numérique)

Découvrir la revue

Citer cet article

Wargon, S. T. (1989). Statistiques officielles et évolution des valeurs sociales : le cas de la personne repère dans les recensements canadiens de 1981 et de 1986. Cahiers québécois de démographie, 18(2), 343-360.

https://doi.org/10.7202/010020ar

\section{Résumé de l'article}

L'évolution des valeurs sociales a entraîné certaines modifications de termes, de concepts et de questions dans les recensements du Canada de 1981 et de 1986. Ce texte présente les changements effectués et leurs conséquences pour certaines données relatives aux ménages et aux familles, en particulier celles qui concernent la personne repère, le soutien du ménageet la classification des familles selon le genre. Dans l'ensemble, l'ajustement à la réalité sociale n'a pas entraîné de ruptures significatives dans les séries chronologiques; il en est résulté des données de meilleure qualité, ainsi que quelques conséquences imprévues, qui posent de nouveaux défis aux recenseurs. 
Cahiers québécois de démographie

Vol. 18, no 2, automne 1989

\title{
Statistiques officielles et évolution des valeurs sociales : le cas de la personne repère dans les recensements canadiens de 1981 et de 1986
}

\author{
Sylvia T. WARGON*
}

Le Canada procède depuis 1871 à un recensement de la population à l'année 1 de chaque décennie. Le recensement quinquennal, introduit en 1906 pour les provinces des Prairies, a été étendu à l'ensemble du pays en 1956. Bien que prescrit en vue d'établir la représentation locale à la Chambre des communes, le recensement répond également au besoin qu'a le gouvernement d'appuyer sa gestion des affaires de l'État sur une information statistique coherrente concernant la population et les ressources (Canada, 1924, xi).

Avec les années, le recensement canadien s'est modifié sous la pression des transformations sociales, économiques et technologiques et pour tenir compte de l'avancement des méthodes d'enquête. Cette évolution a touché le contenu du questionnaire, les modes de recensement de la population et les techniques de dépouillement. En ce qui concerne la collecte, on a instauré, entre autres choses, l'échantillonnage et l'autodénombrement. Le questionnaire lui-même a été adapté à l'évolution de la société canadienne (changements d'attitudes, nouvelles façons de voir, transformation des comportements); des questions ont été éliminées, reformulées, ajoutées... Ces modifications ont forcément eu des effets sur le produit le plus important du recensement : les statistiques.

* Statistique Canada, Direction de la statistique démographique et du recensement, Ottawa. Lauteur, et non Statistique Canada, assume la responsabilitê pleine et entière des idées exprimées dans ce texte. Remerciements à R. Costa, Y. Lavoie, J. Perreault, E. Pryor et M. Sheridan, ainsi qu'aux lecteurs anonymes, dont les suggestions ont êté appréciées. 
Durant les années soixante-dix, l'utilisation du terme chef de ménage (ou de termes similaires) dans les recensements nationaux pour désigner la personne clé dans le ménage et le logement, soit la personne repère selon le jargon technique, a été débattue à l'échelle internationale. Il en est résulté que le Canada, à l'instar d'autres pays comme les États-Unis, la Grande-Bretagne, la France et l'Espagne, a abandonné la désignation traditionnelle de la personne repère.

En 1981, la question 2 du recensement du Canada invitait les membres du ménage à indiquer leur "lien avec la Personne 1". Une question supplémentaire visait à identifier de manière explicite la personne responsable des paiements du ménage. Le recensement quinquennal de 1986 allait dans le même sens que celui de 1981 , à ceci près que la question sur le responsable des paiements a été légèrement remaniée.

L'introduction de ces modifications en 1981 représentait un défi considérable et entrainait beaucoup de travail pour les responsables du recensement. Il a fallu consulter des experts canadiens et étrangers. Il n'êtait pas facile de choisir, pour désigner la personne repère, un nouveau terme à la fois approprié au formulaire et compréhensible à travers le pays. On devait aussi convaincre les utilisateurs des résultats que ces changements étaient nêcessaires et n'allaient pas nuire aux données chronologiques.

Dans les lignes qui suivent, nous allons souligner quelques aspects importants des révisions touchant le contenu du questionnaire et en illustrer les conséquences à partir des données sur les ménages privés. Après avoir examiné les changements apportês aux concepts et à la terminologie, nous en analysons l'impact attendu aussi bien qu'imprêvu sur les résultats. On verra que, dans ce cas, l'ajustement du questionnaire à la réalité sociale n'entrave pas les études exigeant des données chronologiques continues, et que les conséquences non prévues posent des dêfis nouveaux aux responsables des recensements à venir.

\section{CHANGEMENT DES VALEURS SOCIALES ET RECENSEMENT CANADIEN DE 1981}

Au Canada, ce sont des transformations sociales profondes, en cours depuis la Deuxième Guerre mondiale, qui ont conduit à l'adoption du terme Personne 1 et à l'ajout de la question sur le 
responsable des paiements du ménage. La participation accrue des femmes, épouses et mères, au marché du travail, le progrès de la notion d'égalité des sexes et la diversification des modes de vie familiale et individuelle sont des réalités que les Canadiens acceptent de mieux en mieux. Dans ce contexte. divers groupes ont fait valoir que l'appellation chef de ménage (ou celle de chef de famille) avait une connotation d'autant plus sexiste que, jusqu'en 1971 inclusivement, elle devait désigner le mari plutôt que la femme si les deux époux vivaient ensemble.

Il faut souligner que la mobilisation de plus en plus forte contre le terme chef de ménage que l'on a observée durant les années soixante-dix n'est pas le fruit du hasard. Le recensement de 1971 avait introduit l'autodênombrement, procêdure qui amène les membres des ménages à lire, comprendre et interpréter eux-mémes définitions et instructions avant de répondre aux questions.

Les deux concessions faites en 1976, soit la possibilité de déclarer le mari ou la femme comme chef de ménage et l'êlimination du terme chef de famille dans tous les tableaux sur les familles, n'ont pas mis fin aux pressions pour l'abolition de ce terme.

Le fait de désigner un chef, soulignait-on, implique l'existence, au sein des ménages, de relations d'autorité, de domination et de surbordination qui sont mal acceptées dans la société canadienne actuelle. Un quotidien proclamait même : "Today's Family Doesn't Have a Boss" (Toronto Star, 4 mai 1976).

Indêpendamment de ces considérations, il convenait d'améliorer l'information concernant le soutien du ménage et du logement. Il était depuis longtemps devenu évident que ceux qui devaient être désignés comme chefs des ménages n'en étaient pas toujours les soutiens économiques. Il va de soi qu'à plus forte raison, il n'y avait aucune possibilité de savoir si les soutiens du ménage habitaient dans le même logement ou ailleurs. Il était également apparu que les chefs de ménage n'étaient pas toujours les personnes les plus aptes à répondre adéquatement aux questions relatives au logement.

\section{LES REVISIONS DE 1981 ET DE 1986}

Avant 1981, l'étude des familles reposait sur la connaissance du lien de chaque membre du ménage avec la personne 
repère, le chef de ménage. L'apparentement au chef permettait de constituer les familles, de distinguer les membres de la famille et les non-membres partageant le même logement, de constater la composition démographique des ménages, et de départager les familles ou les personnes selon qu'elles maintenaient le logement qu'elles habitaient ou non. Le passage du concept de chef de ménage à celui de Personne 1 a rendu nécessaire, pour atteindre les mêmes objectifs, l'ajout de la question identifiant la personne responsable des paiements du ménage.

1. Écrivez le NOM des résidents habituels de ce logement, au ler juin 1976 :

a) qui sont présents dans le logement,

b) ou temporairement absents (en voyage d'affaires, aux études, à l'hôpital, etc.)

Comptez les personnes sans autre résidence.

1 re PERSONNE

Nom de famille

Prénoms (soulignez le prénom usuel)

2e PERSONNE

Nom de famille

Prénoms (soulignez le prénom usuel]

\section{3e PERSONNE}

Nom de famille

Prénoms (soulignez le prénom usuel)
2. LIEN AVEC LE CHEF DE MÉNAGE Le CHEF de ménage est : l'êpoux OU l'épouse; le père ou la mère, s'il n'y en a qu'un des deux, avec des enfants non mariès; ou tout membre d'un groupe se partageant un logement à parts égales. "Autre" : petit-fils ou petite-fille, oncle, tante, neveu, nièce, beau-père, bellemère, beau-frère, belle-soeur, gendre, bru, employé, partenaire, chambreur. épouse de liemployé, fils du chambreur, etc.

- CHEF de ménage

- Fils ou fille du chef

- Père ou mère du chef

- Frère ou soeur du chef

- Autre (précisez)

- Épouse ou époux du chef

- Fils ou fille du chef

- Père ou mère du chef

- Frère ou soeur du chef

- Autre (précisez)

- Fils ou fille du chef

- Père ou mère du chef

- Frère ou soeur du chef

- Autre (précisez)

Source : formulaire du recensement de 1976, p. 2, questions 1 et 2 (la suite des deux questions concerne les personnes de rang plus élevé, pour quí la formulation est la même que pour la personne 3 ).

Figure 1 - gUESTIONS 1 ET 2 DU RECENSEMENT CANADIEN DE 1976

Les figures 1 et 2 permettent de constater les modifications substantielles apportées à la question 2 . La personne 1 , en tant que personne repère, en 1981 et également en 1986, est simplement le membre adulte du ménage qui, selon des critères déterminés (voir ci-après), a choisi de se déclarer le premier sur le formulaire ou a étê choisi pour ce faire (figure 2). Dès lors, les autres membres sont identifiés, à la question 2, en fonction du lien qu'ils ont avec cette Personne 1 par le sang, par alliance, 
par adoption ou par union libre, ou en fonction de l'absence de tels liens (chambreurs, employés, etc.). Naturellement, l'unique membre des ménages d'une personne est par définition la Personne 1 de son ménage.

1. NOM

Lisez les instructions données à gauche puis écrivez ci-dessous le nom de tous les rêsidents habituels de ce logement le mercredi 3 juin 1981 .

Personne 1

Nom de famille Prénom

Personne 2 Nom de famille Prénom

Personne 3

Nom de famille

Prénom

\section{LIEN AVEC IAA PERSONNE !}

Pour chaque personne du ménage, cochez une seule case pour indiquer le lien avec la Personne 1.

Si vous cochez la case "Autre personne apparentée" ou "Autre personne non apparentée", précisez le lien avec la Personne 1. Voici des exemples d'autres personnes : grand-mère, oncle, fille de la compagne d'appartement, époux de l'employée.

- Personne 1

- Épouse ou époux de la Persorne 1

- Partenaire en union libre de la Personne 1

- Fils ou fille de la Personne 1

- Père ou mère de la Personne 1

- Frère ou soeur de la Personne 1

- Gendre ou bru de la Personne 1

- Beau-père ou belle-mère de la Personne 1

- Beau-frère ou belle-soeur de la Personne 1

- Petit-fils ou petite-fille de la Personne 1

- Neveu ou nièce de la Personne 1

- Autre personne apparentée à la Pers. 1 (précisez)

- Chambreur ou chambreuse

- Époux de la chambreuse ou épouse du chambreur

- Fils ou fille du chambreur ou de la chambreuse

- Compagnon ou compagne d'appartement

- Employé(e)

- Autre personne non apparentéc (précisez)

- Fils ou fille de la Personne 1

- Père ou mère de la Personne 1

- Frère ou soeur de la Personne 1

- Gendre ou bru de la Personne 1

- Beau-père ou belle-mère de la Personne 1

- Beau-frère ou belle-soeur de la Personne 1

- Petit-fils ou petite-fille de la Personne 1

- Neveu ou nièce de la Personne 1

- Autre personne apparentée à la Pers. 1 (précisez)

- Chambreur ou chambreuse

- Époux de la chambreuse ou épouse du chambreur

- Fils ou fille du chambreur ou de la chambreuse

- Compagnon ou compagne d'appartement

- Employé(e)

- Autre personne non apparentée (précisez)

Source : formulaire du recensement de 1981, p. 2 (la suite des deux questions concerne les personnes de rang plus élevé, pour qui la formulation est la même que pour la personne 3). 
Question 7 (1981)

Inscrivez le nom de la personne (ou de l'une des personnes) qui vit ici et qui est responsable du paiement du loyer, ou de l'hypothèque, ou des taxes, ou de l'électricité, etc., pour ce logement.

Nom :

Prénom :

Cette personne devrait répondre aux questions suivantes sur ce logement.

NOTA : Si aucune personne vivant ici n'effectue de tels paiements, cochez ici et rẻpondez vous-même aux questions sur ce logement.

Source : formulaire du recensement de 1981, p. 4.

Question 8 (1986)

a) Inscrivez le nom de la personne (ou de l'une des personnes) qui vit ici et qui est responsable du paiement du loyer, ou de l'hypothèque, ou des taxes, de l'électricité, etc., pour ce logement.

Nom :

Prénom :

Cette personne deura répondre aux questions 8 b) à 13.

NOTA : Si aucune personne vivant ici n'effectue de tels paiements, cochez ici et répondez vous-même aux questions $8 b)$ à 13.

Source : formulaire du recensement de 1986, p. 4.

Figure 3 - gUESTIONS 7 (1981) ET 8 (1986) DU RECENSEMENT CANADIEN

La figure 3 présente la question additionnelle ( 7 en 1981,8 en 1986) qui identifie le soutien économique du logement et permet de mesurer la fréquence des cas où ce soutien diffère de la personne repère. De plus, elle vise à connaitre les ménages dont le responsable des paiements ne fait pas partie. Antérieurement, aucune information de cette nature n'était disponible dans les recensements canadiens.

\section{DU "CHEF DE MÉNAGE " À LA " PERSONNE 1 " : CRITÉRES DE SELLCTION}

Auparavant, en 1976, le changement s'était limité à permettre soit au mari, soit à la femme de se déclarer chef de ménage. En 1981, le terme chef de ménage a été remplacé par celui de Personne 1. Mais les critères de sélection de la personne repère demeurent ceux de 1976 à ceci près qu'on mentionne explicitement que dans le cas de couples non mariês, la Personne 1 peut être l'un ou l'autre des partenaires (figure 4). Les critères appliqués en 1986 sont ceux de 1981.

Le traitement des unions libres dans la sélection de la personne repère fournit un exemple intéressant de la prise en compte de l'êvolution des valeurs sociales : le recensement de 1971 est muet en ce qui concerne ces unions, à l'exception d'une 
- Au recensement de 1986, la personne repère (Personne l) devait être choisie de la même manière qu'à celui de 1981 .

- Au recensement de 1981, l'expression * Personne 1 a remplacé celle de "chef de ménage" pour désigner la personne repère du ménage. La Personne I devait être choisie de la façon suivante :

- l'un des conjoints (époux ou épouse) d'un couple marié vivant dans le même logement;

- l'un ou l'autre des partenaires vivant en union libre;

- le père ou la mère, lorsque seulement un des deux vit avec son ou ses enfants célibataires (jamais mariés) quel que soit leur âge; ou encore,

- tout adulte du ménage.

- En. 1976, le chef de ménage était :

- l'époux ou l'épouse;

- le père ou la mère, lorsque seulement un des deux vivait avec ses enfants célibataires (jamais mariés);

- tout membre d'un groupe partageant un logement à parts égales.

- En 1971, le chef de ménage était :

- l'époux plutôt que l'épouse;

- le père ou la mère, lorsque seulement un des deux vivait avec ses enfants célibataires (jamais mariés):

- tout membre d'un groupe partageant un logernent à parts égales.

Source : Canada, 1987a.

\section{Figure 4-CRITÈRES DE SÉLECTION DE LA PERSONNE REPÈRE DANS LES RECENSEMENTS DU CANADA, 1971-1986}

instruction du guide invitant les partenaires qui vivent en union libre à se déclarer mariés. Ces unions sont comprises dans la catégorie "époux-épouse "dans les données de 1971 et de 1976 relatives aux familles. En 1981, on l'a vu, les partenaires vivant en union libre sont mentionnés explicitement dans les critères de sélection de la personne repère, et en 1986 les unions libres forment une catégorie distincte dans les publications du recensement sur les familles.

\section{DU "CHEF DE MÉNAGE " À LA " PERSONNE RESPONSABLE DES PAIEMENTS DU MÉNAGE " : CRITÈRES DE SÉLECTION}

Avant 1981, on tenait simplement pour acquis que le chef du ménage (et de la famille) en était le soutien économique, même si de toute évidence ce n'était souvent pas le cas. Le chef de ménage n'est décrit comme le soutien économique du ménage qu'en 1951 (Sametz et Pleizier, 1980: 11). Notons que la responsabilité des paiements n'apparaît pas dans les critères de sélection du chef dans le recensement de 1951 ni dans les recensements postérieurs ( voir la figure 4). 
En 1981, la question 7 demande aux répondants d'inscrire le nom de la personne ou d'une des personnes qui vit dans le logement et est responsable du paiement du loyer, ou de l'hypothèque, ou des taxes, ou de l'électricité, etc. Les critères de sélection sont inclus dans la question elle-même, et une instruction stipule que cette personne devra répondre aux questions sur le logement. Une case-réponse placée à la suite de cette instruction permet au répondant, le cas échéant, d'indiquer que la personne qui effectue les paiements ne réside pas dans le logement, et il est priê de rêpondre aux questions relatives à celui-ci. En 1986, la question 8, avec le minimum de changements dans la formulation et la disposition, collecte la même information (figure 3).

Comment la nouvelle question modifie-t-elle les concepts de famille principale et de famille secondaire en 1981 et en 1986 ? Dans les recensements antérieurs, la famille principale, c'est-à-dire qui est considérée comme responsable de l'entretien du ménage et du logement, était celle du chef de ménage. Toute autre farnille vivant dans le même logement était automatiquement désignée comme secondaire. En 1981 et en 1986, est considérée comme famille principale celle dont fait partie la personne responsable des paiements du ménage, et comme secondaire toute famille dont cette personne ne fait pas partie.

Certains points étaient fréquemment soulevées par les utilisateurs de données. Les questions posées en 1981 et en 1986 sont-elles appropriées pour déterminer qui paie les frais relatifs au logement et à l'entretien du ménage ? Pourquoi n'identifier qu'un responsable des paiements alors qu'on peut bien penser que les membres adultes du ménage ou que les familles qui corésident partagent divers coûts? N'est-il pas aussi possible que le répondant indique la personne qui tient les comptes du ménage, qui voit à ce que les paiements soient effectuées dans les délais, etc. ?

On doit comprendre que les responsables du recensement sont sensibles à ce type de remarques et en reconnaissent la pertinence. Néanmoins, il leur fallait satisfaire à certaines exigences : la nouvelle question devait être directe et formulée de manière à être comprise partout au pays; il fallait aussi qu'elle aille chercher l'information concernant les paiements relatifs au ménage auprès d'une personne compétente. En consultant la figure 3 , on verra que la formulation de la question 7 (ou 8 en 1986) ne rejette pas plus la possibilité du partage des coûts qu'elle n'explicite la portée du terme 
responsable. Cependant, le guide de 1986 précise : "si plus d'une personne est responsable des paiements, inscrivez le nom de la personne qui verse habituellement le plus gros montant * .

\section{EFFETS DE CES REVISIONS}

On savait que les révisions de certains concepts et de la terminologie et l'addition de certaines questions, dêcrites cidessus, auraient un impact sur les résultats du recensement, mais on pouvait mal en supputer l'ampleur. On s'est donc préoccupé de certains effets des révisions de façon à répondre tant aux utilisateurs de sêries chronologiques qui s'opposent à toute solution de continuité qu'aux partisans de réformes permettant au recensement de s'ajuster à la réalité sociale.

Idéalement, pour mesurer la nature et l'ampleur de l'impact des révisions sur les statistiques concernant les ménages et les familles, il faudrait déterminer, par comparaison avec les donnêes antérieures correspondantes, la part imputable à la modification des concepts et de la terminologie, la part due aux méthodes de collecte et de traitement des données, et enfin la part liêe aux transformations d'ordre économique, démographique ou social. Les contraintes de temps et d'espace ne nous permettent pas de prēsenter une analyse aussi poussée.

En lieu et place, nous allons évaluer l'impact des révisions sur les statistiques relatives au genre de familles (principales ou secondaires), à la personne repère et au soutien du ménage. Nous présentons également des données qui montrent pour la première fois le degré de concordance, en 1981 et en 1986, entre la personne repère et le soutien économique du ménage.

\section{L'IMPACT ATTENDU DES REVISIONS DES RECENSEMENTS DE 1981 ET DE 1986}

\section{L'impact sur les statistiques relatives au genre de familles}

Les données des recensements révèlent que la proportion des familles principales est passée de $90 \%$ à $97 \%$ de 1951 à 1986 (tableau 1). L'examen de la série chronologique montre que l'incorporation des changements n'a entraîné qu'un léger décalage en 1981 et a produit des résultats de meilleure qualité.

L'accroissement des familles principales, constant de 1951 à 1976, traduit la propension des familles nucléaires à occuper 
TABLEAU 1

Familles de recensement dans les ménages privés selon le genre, Canada, 1951-1986

\begin{tabular}{|c|c|c|c|c|c|c|}
\hline \multirow{2}{*}{ Familles } & \multicolumn{2}{|c|}{ Principales } & \multicolumn{2}{|c|}{ Secondaires } & \multicolumn{2}{|c|}{ Toutes } \\
\hline & $\mathbf{N}$ & $\%$ & $\mathbf{N}$ & $\%$ & $\mathbf{N}$ & $\%$ \\
\hline 1951 & 2966738 & 90,2 & 320645 & 9,8 & 3287384 & 100,0 \\
\hline 1956 & 3425890 & 92,3 & 285610 & 7,7 & 3711500 & 100,0 \\
\hline 1961 & 3911529 & 94,3 & 235915 & 5,7 & 4147444 & 100,0 \\
\hline 1966 & 4345718 & 96,0 & 180548 & 4,0 & 4526266 & 100,0 \\
\hline 1971 & 4898290 & 96,6 & 172390 & 3,4 & 5070685 & 100,0 \\
\hline 1976 & 5604950 & 97,8 & 122940 & 2,2 & 5727895 & 100,0 \\
\hline $1981^{a}$ & 6133205 & 97,0 & 191770 & 3,0 & 6324975 & 100,0 \\
\hline $1986^{a}$ & 6534365 & 97,0 & 200610 & 3,0 & 6734980 & 100,0 \\
\hline
\end{tabular}

Sources : Recensements du Canada de 1957 à 1986, données publićes ou inédites.

a. Catégorie résiduelle : $61945(1,0 \%)$ en 1981 et $56975(0,8 \%)$ en 1986; familles incluses dans la catégorie Familles secondaires à $98 \%$.

seules leur logement. On s'attendait, au moment de la planification du recensement de 1981 , à ce que la proportion des familles principales continue à progresser. Pourtant, l'analyse des données chronologiques avait, dans les années soixante-dix, amené les chercheurs (qui anticipaient la crise économique) à envisager un ralentissement de la tendance.

Certains facteurs indépendants de l'évolution du phénomène lul-même pouvaient également influer sur les résultats de 1981. Ainsi, la très faible proportion atteinte par les familles secondaires en $1976(2,2 \%)$ laissait peu de place à la poursuite du déclin. On connaît également la sensibilité des petits nombres à des facteurs tels que l'amélioration de la couverture du recensement ou des modes de collecte des données, les variations de la qualité des réponses ou de l'erreur liêe au traitement manuel ou informatique, etc. Même faible, le poids des changements de cette nature peut infléchir les tendances ou les renverser lorsqu'on considère des sousgroupes de petite taille.

Le mince accroissement du nombre et de la proportion des familles secondaires de 1976 à 1981 (tableau 1) est principalement imputable au fait qu'environ $98 \%$ des quelque 62000 familles d'une catégorie "résiduelle" ont été plus adéquatement classées en 1981 qu'auparavant. Puisqu'elles habitaient des logements dans lesquels ne rësidait pas le soutien économique du ménage, ce sont des familles secondaires que, faute d'information suffisante, on rangeait 
antérieurement dans la catégorie "familles principales". Quand on ajuste les données de 1981 pour tenir compte de cette reclassification, on constate qu'il y a une relative stabilité dans l'évolution des familles secondaires entre 1976 et 1981.

\section{Limpact sur la personne repère}

On avait espéré qu'en substituant le terme neutre Personne 1 à celui de chef de ménage, on réduirait les réticences des répondants et que, par conséquent, on obtiendrait des données plus justes sur les transformations touchant la formation et la composition des ménages. L'expêrience semble dêmontrer que ces attentes se sont concrétisêes sans solution de continuité en dépit des craintes exprimées par les utilisateurs de données chronologiques. Plus encore, les projections officielles des ménages et des familles de Statistique Canada ont remplacé le concept de chef de ménage par celui de soutien du ménage (Canada, 1987b).

Les tableaux 2 et 3 et la figure 5 illustrent l'impact des modifications du formulaire sur les informations relatives à la personne repère. Quelques explications sont nécessaires pour la compréhension de ces données. Ainsi, après 1976, aucun tableau du recensement n'a portê sur les caractéristiques de la personne repère du ménage. On en a d'ailleurs déconseillé l'analyse pour des raisons généralement admises par les utilisateurs avertis. En effet, la désignation d'une personne

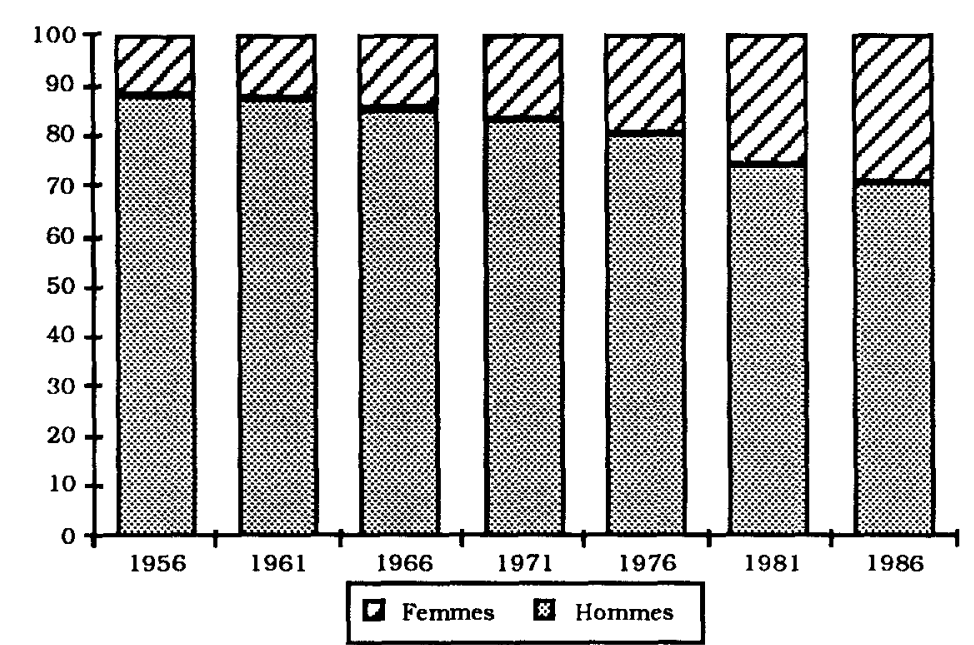

Figure 5 - PERSONNE REPERE, SELON LE SEXE, CANADA, 1956-1986 


\section{TABLEAU 2}

Distribution des personnes repères dans les ménages privés selon leur situation dans la famille de recensement et selon le sexe, 1976, 1981 et 1986 (\%)

\begin{tabular}{lccc}
\hline & $\begin{array}{c}1976 \\
\text { Chef de ménage }\end{array}$ & $\begin{array}{c}1981 \\
\text { Personne }\end{array}$ & $\begin{array}{c}1986 \\
\text { Personne 1 }\end{array}$ \\
\hline Membres d'une famille & 78,2 & 74,9 & 73,4 \\
$\quad$ Hommes & 71,1 & 63,3 & 59,2 \\
Femmes & 7,1 & 11,5 & 14,2 \\
Personnes hors-famille & 21,8 & 25,1 & 26,6 \\
$\quad$ Hommes & 9,1 & 10,7 & 11,3 \\
$\quad$ Femmes & 12,7 & 14,4 & 15,3 \\
Total hommes & 80,2 & 74,1 & 70,5 \\
Total femmes & 19,8 & 25,9 & 29,5 \\
Effectif des & & & \\
personnes repères & 7166905 & 8281530 & 8991670 \\
$\quad$ (\%) & 100,0 & 100,0 & 100,0 \\
\hline
\end{tabular}

Source : Recensements du Canada de 1976, 1981 et 1986. Données publiées et inédites.

repère étant un artifice purement arbitraire destiné à faciliter l'énumération des ménages, les données concernant cette personne sont discutables et n'ont pas de réelle signification.

À l'opposê, des démographes et d'autres spêcialistes des sciences sociales ont fait valoir que les séries chronologiques sur les caractéristiques de base des personnes repères, tels l'âge, le sexe, l'état matrimonial et le statut dans la famille, renseignent sur la dynamique de la formation et de la composition des ménages et sur les transformations des modes de vie (Kobrin, 1973; Duchesne, 1987), et servent au calcul des taux de chefs nécessaires aux projections. En fait, l'examen de l'impact des changements de 1981 et de 1986 sur les donnêes relatives à la personne repère s'impose, vu l'utilisation continue qui en a étê faite dans la projection des ménages et des familles.

On constate, dans le cas de la personne repère comme dans celui du genre de famille, que les données de 1981 et de 1986 s'inscrivent dans la longue tendance. Les révisions apportées ces années-là n'ont donc pas causé de rupture majeure ou engendrê d'inefficacité. Les tendances qui se manifestent sont connues. L'accroissement du nombre de femmes qui se désignent comme personnes repères témoigne de la plus forte présence féminine dans la main-d'oeuvre et de la hausse rapide 
TABLEAU 3

Personnes repères dans les ménages privés par âge et par sexe, Canada, 1976, 1981 et 1986

\begin{tabular}{|c|c|c|c|c|c|c|}
\hline \multirow{2}{*}{$\begin{array}{l}\text { Groupes } \\
\text { d'âge et } \\
\text { sexe }\end{array}$} & \multicolumn{2}{|c|}{$\begin{array}{c}1976 \\
\text { Chef de ménage }\end{array}$} & \multicolumn{2}{|c|}{$\begin{array}{c}1981 \\
\text { Personne } 1\end{array}$} & \multicolumn{2}{|c|}{$\begin{array}{c}1986 \\
\text { Personne } 1\end{array}$} \\
\hline & $\mathbf{N}$ & $\%$ & $\mathbf{N}$ & $\%$ & $\mathbf{N}$ & $\%$ \\
\hline $\begin{array}{c}\text { Total } \\
\text { H } \\
\text { F }\end{array}$ & $\begin{array}{l}5750225 \\
1415870\end{array}$ & $\begin{array}{l}80 \\
19\end{array}$ & & $\begin{array}{r}100,0 \\
74,1 \\
25,9\end{array}$ & & $\begin{array}{r}100,0 \\
70,5 \\
29,5\end{array}$ \\
\hline $\begin{array}{c}15-24(\mathrm{~T}) \\
\mathrm{H} \\
\mathrm{F}\end{array}$ & & $\begin{array}{r}100 \\
73 \\
26\end{array}$ & & $\begin{array}{r}100,0 \\
62,3 \\
37,7\end{array}$ & $\begin{array}{l}540160 \\
295730 \\
244,430\end{array}$ & $\begin{array}{r}100,0 \\
54,7 \\
45,3\end{array}$ \\
\hline $\begin{array}{c}25-34(\mathrm{~T}) \\
\mathrm{H} \\
\mathrm{F}\end{array}$ & & $\begin{array}{r}100 \\
86 \\
13\end{array}$ & & $\begin{array}{r}100,0 \\
77,5 \\
22,5\end{array}$ & & $\begin{array}{r}100 \\
7 \\
2\end{array}$ \\
\hline $\begin{array}{c}35-44(\mathrm{~T}) \\
\mathrm{H} \\
\mathrm{F}\end{array}$ & $\begin{array}{r}133 \\
118 \\
15\end{array}$ & $\begin{array}{r}100 \\
88 \\
11\end{array}$ & & $\begin{array}{r}100,0 \\
82,0 \\
18,0\end{array}$ & & $\begin{array}{r}100 \\
77 \\
22\end{array}$ \\
\hline $\begin{array}{c}45-54(\mathrm{~T}) \\
\mathrm{H} \\
\mathrm{F}\end{array}$ & $\begin{array}{r}1305655 \\
1115725 \\
189930\end{array}$ & $\begin{array}{r}100,0 \\
85,5 \\
14,5\end{array}$ & $\begin{array}{r}1364435 \\
1111230 \\
253205\end{array}$ & $\begin{array}{r}100,0 \\
81,4 \\
18,6\end{array}$ & $\begin{array}{r}1407330 \\
1109685 \\
297650\end{array}$ & $\begin{array}{r}100,0 \\
78,9 \\
21,1\end{array}$ \\
\hline $\begin{array}{c}55-64(\mathrm{~T}) \\
\mathrm{H} \\
\mathrm{F}\end{array}$ & $\begin{array}{r}1079000 \\
841245 \\
237755\end{array}$ & $\begin{array}{r}100,0 \\
78,0 \\
22,0\end{array}$ & $\begin{array}{r}1220860 \\
913555 \\
307305\end{array}$ & $\begin{array}{r}100,0 \\
74,8 \\
25,2\end{array}$ & $\begin{array}{r}1334270 \\
986265 \\
348005\end{array}$ & $\begin{array}{r}100,0 \\
73,9 \\
26,1\end{array}$ \\
\hline $\begin{array}{c}65+(T) \\
H \\
F\end{array}$ & $\begin{array}{r}1178775 \\
720380 \\
458395\end{array}$ & $\begin{array}{r}100,0 \\
61,1 \\
38,9\end{array}$ & $\begin{array}{r}1423165 \\
829185 \\
593975\end{array}$ & $\begin{array}{r}100,0 \\
58,3 \\
41,7\end{array}$ & $\begin{array}{r}1647925 \\
935500 \\
712425\end{array}$ & $\begin{array}{r}100,0 \\
56,8 \\
43,2\end{array}$ \\
\hline
\end{tabular}

Source : Recensements du Canada, 1976, 1981 et 1986. Données publiées ou inédites. Note : Tous les chiffres se terminent par 5 ou 0 à cause de l'arrondissement aléatoire.

de la monoparentalité (tableau 2). L'augmentation est en effet plus marquée chez les épouses et les mères sans conjoint, dont la part a doublé, passant de 7,1 \% en 1976 à $14,2 \%$ en 1986 .

La progression du pourcentage de jeunes femmes qui se déclarent personnes repères attire l'attention (tableau 3); les jeunes générations sont à l'avant-garde des changements de comportements. L'évolution est sensible chez les jeunes adultes de 15 à 24 ans. Chez les 25-34 ans et les 35-44 ans, les proportions ont doublé et les nombres absolus ont presque triplé. On remarque également la propension croissante des femmes âgées à former des ménages non familiaux d'une seule personne; cette tendance ressortait déjà nettement des statistiques des années soixante-dix. 
TABLEAU 4

Soutien du ménage selon le sexe, ménages privés, Canada, 1976, 1981 et 1986

\begin{tabular}{lcccccc}
\hline & \multicolumn{2}{c}{ Tous } & \multicolumn{2}{c}{ Hommes } & \multicolumn{2}{c}{ Femmes } \\
& $\mathrm{N}$ & $\%$ & $\mathrm{~N}$ & $\%$ & $\mathrm{~N}$ & $\%$ \\
\hline $\begin{array}{l}\text { 1976, chef } \\
\text { de ménage }\end{array}$ & 7166095 & 100,0 & 5750220 & 80,2 & 1415870 & 19,8 \\
$\begin{array}{l}\text { 1981, soutien } \\
\text { de ménage }\end{array}$ & 8281530 & 100,0 & 6179555 & 74,6 & 2101970 & 25,4 \\
$\begin{array}{l}\text { 1986, soutien } \\
\text { de ménage }\end{array}$ & 8991670 & 100,0 & 6436825 & 71,6 & 2554825 & 28,4 \\
\hline
\end{tabular}

Source : Recensement du Canada, 1976, 1981 et 1986, données publiêes.

Note : tous les chiffres se terminent par 5 ou 0 à cause de l'arrondissement aléatoire.

La figure 5 permet aussi de constater que les révisions de 1981 et de 1986 n'ont introduit ni rupture ni distorsion dans la tendance des femmes à occuper le statut de personne repère. Mais on se demande souvent pourquol au moins $50 \%$ de femmes ne sont pas désignées comme Personne 1. La dêcision d'un grand nombre de couples de mentionner le mari en première place illustre sans doute un phênomène bien connu en sociologie : la persistance des modèles traditionnels alors que de nouvelles valeurs se sont imposées dans les faits.

Les données de 1981 et de 1986 (tableau 4) confirment que la proportion des femmes qui se déclarent soutien économique du ménage croît constamment depuis 1976. Mais, comme c'était le cas pour le statut de Personne 1, et pour les mêmes raisons sans doute, beaucoup moins de $50 \%$ des femmes sont désignées comme personnes responsables des paiements du logement où elles rêsident. Il se peut aussi que cette façon de rêpondre traduise " une certaine division sexuelle des deppenses, division qui fait en sorte que les revenus des femmes (mariées ou en union libre) servent généralement à couvrir les dépenses du ménage liées à l'alimentation, à l'habillement et aux services plutôt que celles liées à l'habitation. Une définition qui ne retient que les dépenses liées au logement conduit à dêsigner plus souvent les hommes que les femmes comme soutien du ménage, et ce, malgré le fait qu'ils contribuent pour une part plus faible aux autres dépenses du ménage (Marcoux et Mongeau, $1989: 131$ ). Notons que les questions 7 et 8 (figure 3) parlent des coûts "de ce logement ": mais les dictionnaires parlent des coûts " du ménage " et du " soutien du ménage ". 


\section{L'information inédite sur la Personne 1 et sur la personne responsable des paiements du ménage}

On doit aux changements introduits dans les recensements de 1981 et de 1986 la possibilité de mesurer, pour la première fois, la correspondance entre la Personne 1 et celle qui est responsable des paiements du ménage (" soutien ").

Comme on s'y attendait, dans plus de $90 \%$ des cas, la même personne cumule les deux statuts. Toutefois, en 1986, la proportion des cas de non-concordance a progressé $(7,9 \%$ des cas) par rapport à 1981 (6,8\% des cas) (tableau 5).

\section{TABLEAU 5}

Information inédite concernant la Personne 1 (P 1) et la personne responsable des paiements du ménage (PRPM), Canada, 1976 a, 1981 et 1986

\begin{tabular}{|c|c|c|c|c|}
\hline \multirow[b]{2}{*}{ Ménages... } & \multicolumn{2}{|c|}{1981} & \multicolumn{2}{|c|}{1986} \\
\hline & $\mathrm{N}$ & $\%$ & $\mathbf{N}$ & $\%$ \\
\hline Privés ${ }^{b}$ & 8281530 & 100,00 & 8991675 & 100,0 \\
\hline Où $P 1=P R P M$ & 7717760 & 93,2 & 8281095 & 92,1 \\
\hline Où P I $1 \neq \mathrm{PRPM}$ & 563770 & 6,8 & 710580 & 7,9 \\
\hline $\begin{array}{l}\text { Comprenant } \\
\text { PRPM et P } 1\end{array}$ & 450100 & 5,4 & 620225 & 6,9 \\
\hline $\begin{array}{l}\text { Où PRPM est le } \\
\text { conjoint de } \mathrm{P} 1\end{array}$ & 357085 & 4,3 & 507480 & 5,6 \\
\hline $\begin{array}{l}\text { Ménage de } P 1 \neq \\
\text { ménage de PRPM }\end{array}$ & 113670 & 1,4 & 90355 & 1,0 \\
\hline
\end{tabular}

Source : Recensement du Canada, 1976. 1981 et 1986, données publiées ou inédites.

a Pour 1976, les données détaillées ne sont pas disponibles. Ménages privés = 7166095 $(100,0 \%)$.

b Le nombre de ménages privés correspond au nombre de Personnes 1.

\section{L'IMPACT NON PRÉVU DES REVISIONS SUR LES REPONSES}

Lorsqu'on a tratté les données des recensements de 1981 et de 1986, on a relevé des conséquences inattendues des modifications des concepts et des questions introduites en 1981.

Ainsi, il est apparu que des couples mariés se sont montrés réticents à désigner une Personne 1 et ont donné des réponses qui exigeaient une opération de "nettoyage " : par exemple, ils 
ont omis de remplir le bloc du questionnaire réservé à la Personne 1 , ont fait une marque dans le bloc qui suit et ont ajouté dans le troisième la mention en clair de "conjoint * de la Personne 1.

De plus, la vérification des données a montré que des couples ont manifestement "choisi" de déclarer l'un des conjoints Personne 1 et l'autre responsable des paiements. En 1981, environ 357000 ménages, soit 6,5\% de tous les ménages époux-épouse unifamiliaux, ont inscrit comme responsable des paiements le conjoint de la Personne 1. En 1986, 507500 ménages, soit $8,8 \%$, ont fait de même. Vraisemblablement, la question introduite en 1981, sans qu'on l'ait prévu, a permis aux couples de signaler de cette manière un partage effectif des responsabilités. Notons que ni le questionnaire de $1981 \mathrm{ni}$ celui de 1986 n'indiquaient explicitement, dans les instructions préliminaires, que les répondants auraient d'abord à identifier une Personne 1 et ensuite un responsable des paiements. En eût-il été ainsi, la proportion des conjoints qui ont divisé ces statuts entre eux aurait pu être plus élevée en 1981 et en 1986.

\section{CONCLUSION}

L'introduction du terme Personne 1 et de la question sur la personne responsable des paiements s'est révélée, en ce qui concerne l'impact attendu, avantageuse pour tous les utilisateurs prêts à accepter l'inconvénient de discontinuités mineures voire négligeables dans les termes et dans les définitions, contre un meilleur ajustement à l'évolution sociale.

Quant à l'impact non prévu, il pose des défis aux responsables des recensements à venir. S'il existe d'excellentes raisons d'identifier le soutien économique du ménage, il paraît indispensable de prendre en compte le fait que plusieurs personnes peuvent se partager cette fonction dans le contexte canadien actuel. Ainsi, des conjoints qui travaillent divisent entre eux les dépenses du ménage. D'autres reconnaissent la valeur du travail domestique, qui constitue une forme de participation au maintien du ménage. La notion de partage est également pertinente dans le cas des ménages non familiaux de plusieurs personnes. Il n'est donc pas étonnant que les pressions en faveur d'une question sur le partage des coûts 
d'entretien du ménage et du logement se multiplient. En réponse, les responsables du recensement canadien ont inclus une question à cet effet dans le test du recensement national effectué en novembre 1988.

Les données recueillies au moyen de cette question et d'une deuxième interrogation des membres des ménages quil $\mathrm{y}$ ont répondu démontrent la possibilité d'obtenir de bons renseignements sur l'extension du partage des coûts relatifs au maintien du logement. Du reste, dans le cadre de la préparation du recensement de 1991, Statistique Canada travaille à la mise au point d'une question de cette nature. Ce partage constitue une donnee sociale qui importe de plus en plus; voilà un autre dêfi que les responsables du recensement auront à relever durant les années quatre-vingt-dix.

\section{RÉFÉRENCES BIBLIOGRAPHIgUES}

CANADA, 1924. Sixth Census of Canada, 1924 (recensement de 1921). Volume I, Number, Sex and Age Distribution. Racial Origins. Religions. Ottawa, Dominion Bureau of Statistics.

CANADA, 1987a. Dictionnaire de référence. Recensement du Canada 1986. Ottawa, Statistique Canada, janvier.

CANADA, 1987b. Projections provisoires des ménages et des familles pour le Canada, les provinces et les territoires jusqu'à 2006. Ottawa. Statistique Canada, janvier.

CANADA, 1951-1986. Census of Canada. All years. Données publiées et données inédites.

DUCHESNE, L. 1987. Les ménages et les familles au Québec. Guébec, Bureau de la statistique du Guébec.

KOBRIN, F. 1973. * Household Headship and its Changes in the United States, 1940-1960, 1970". Journal of the American Statistical Association, 68, 344 (décembre).

MARCOUX, R. et J. MONGEAU, 1989. «Votre ménage a-t-il changé de "boss" entre 1971 et 1981 ? . Cahiers québécois de démographie, 18,1 (printemps), 115-136.

SAMETZ, R. et C. PLEIZIER, 1980. La personne repère à l'intérieur du ménage dans le cadre du recensement du Canada: solutions envisagées et implications. Ottawa, Statistique Canada, avril.

WARGON, S., L. ALBERT et B. HAMM, 1982. Census Families by Family Type: Primary and Secondary Families. 1981 Census. Certification Report. Ottawa, Statistique Canada, mai, miméo.

WARGON, S., J. PERREAULT et R. COSTA, 1987. The Selection of Person 1 and Household Maintainer in Husband-Wife Households: A Joint Recommendation for the 1991 Census from Demography Division and Housing, Family, and Social Statistics Division. Statistique Canada, 28 septembre, miméo. 


\section{RÉSUMÉ - SUMMARY — RESUMEN}

\section{WARGON SYIVIa T. - STATISTIQUES OFFICIELLES ET ÉVOLUTION}

DES VALEURS SOCIALES : LE CAS DE LA PERSONNE REPÈRE DANS LES RECENSEMENTS CANADIENS DE 1981 ET DE 1986

L'évolution des valeurs sociales a entrainé certaines modifications de termes, de concepts et de questions dans les recensements du Canada de 1981 et de 1986. Ce texte présente les changements effectués et leurs conséquences pour certaines données relatives aux ménages et aux familles, en particulier celles qui concernent la personne repère, le soutien du ménage et la classification des familles selon le genre. Dans l'ensemble, l'ajustement à la réalité sociale n'a pas entrainé de ruptures significatives dans les séries chronologiques; il en est résulté des données de meilleure qualité, ainsi que quelques conséquences imprévues, qui posent de nouveaux défis aux recenseurs.

\section{WARGON Sylvia T. - OFFICIAL STATISTICS AND CHANGING SOCIAL VALUES:}

Changing social values brought about modifications in certain concepts, terminology and questions in the 1981 and 1986 Censuses of Canada. This text examines how the term head of household was replaced by another term for household reference person, Person 1, and person responsible for household payments, and the consequences for certain data series. On the whole, these changes did not cause any appreciable break in, but rather improved, relevant statistical series. Some unanticipated results of these changes pose a new challenge for Canada's census takers in the 1990's.

WARGON Sylvia T. - ESTADISTICAS OFICIALES Y EVOLUCIÓN DE LOS VALORES SOCLALES: EL CASO DE LA PERSONA "REFERENCIA" EN LOS CENSOS CANADIENSES DE 1981 Y 1986.

La evolución de los valores sociales ha producido algunas modificactones de términos, de conceptos y de cuestiones en los censos del Canadá de 1981 y de 1986. Este texto presenta los cambios realizados y sus consecuencias para clertos datos relativos a los matrimonios y a las familias, particularmente aquellos que conciernen la persona referencia, el sostén del matrimonio y la clasificación de las familias según el genero. En el conjunto, el ajuste a la realidad social no ha llevado a rupturas significativas en las series cronológicas; se logró así datos de mejor calidad, asi como algunas consecuencias imprevistas, las cuales traen nuevos desafios a los censores. 